\title{
Silicon Microstructures and Microactuators for Compact Computer Disk Drives ${ }^{1}$
}

\author{
Denny K. Miu ${ }^{2}$ and Yu-Chong Tai ${ }^{3}$
}

In 1956, IBM shipped the first magnetic rigid disk drive which was 24 inch in diameter, fitted inside a box the size of an industrial size refrigerator, stored 5 megabytes and sold for tens of thousands of dollars. In 1983, Seagate shipped the first magnetic disk drive for the PC market which was 5-1/4 inch in diameter, fitted in a box half the size of a shoebox, stored also 5 megabytes and sold for less than $\$ 1,500$. In 1992 , HP shipped what is still the world's smallest commercially available rigid disk drive, which is 1.3 inch in diameter, fits in a box the size of a matchbook, stores 40 megabytes and sells for $\$ 150$. In terms of areal storage density and cost per megabyte, this has far exceeded 10 time improvement in 10 years; in fact, since 1991 magnetic recording disk drives have doubled in performance every eighteen months.

It is projected in another five years, the industry will be capable of delivering gigabyte disk drive cartridges suitable for palmtop multimedia personal communicators. At UCLA and Caltech, we believe silicon micromachining technology will play an important role in the fabrication of high-bandwidth servocontrolled miniaturized microelectromechanical components for such super-high-capacity, super-compact computer disk drives. For the past three years we have been collaborating on a number of industry and government supported joint research projects to develop the necessary technology building blocks to significantly increase the performance of drive design of the future. These efforts include one to design and fabricate a silicon read/write head microgimbal with integrated electrical and mechanical interconnects, which targets the next-generation $30 \%$ form factor pico-sliders, and an electromagnetic piggyback

\footnotetext{
1 The work presented here was conducted at the Caltech Micromachining Laboratory with support from the University of California MICRO program and matching funds from the Applied Magnetics, Hewlett-Packard, IBM, Magnacomp, Maxtor, Quantum, Read-Rite and Seagate Technology Corporations, and the National Storage Industry Consortium (NSIC). The results are the combined efforts of our many colleagues, Amish Desai, Wen Hsieh, Raanan Miller, Weilong Tang, Viktoria Temesvary and Shuyun Wu.

${ }^{2}$ Assistant Professor, Mechanical Engineering, University of California, Los Angeles, CA 90024-1597

${ }^{3}$ Assistant Professor, Electrical Engineering, California Institute of Technology, Pasadena, CA 91125
}

planar microactuator for super-high-track-density applications, both of which utilize state-of-the-art silicon micromachining fabrication techniques.

\section{Application Background}

Today's digital magnetic recording techniques provide storage at about 400 to 700 megabits of data per square inch of recording surface. This performance is extremely impressive considering that the areal density of the first commercial product was only 2 kilobits per square inch. Magnetic recording has remained and will continue to remain the most viable option when compared to either optical storage or solid-state memory (including Flash) due to its cost-competitiveness (in both component and media levels), overall recording density, access speed and data rate, reliability and non-volatileness, and its proven ability to adapt to application-specific needs. More importantly, unlike optical recording where the recording density is currently being limited by the wavelength of the illumination source and solid-state memory where density has always been hampered by advances in photolithographic and thermomechanical packaging techniques, recording density of magnetic recording is currently orders of magnitude away from any physical limits.

Figure 1 shows the major electromechanical components of the conventional single-actuator/pivotbearing rigid disk drive design. In computer disk drives digital information is recorded in concentric tracks on rotating disks using miniaturized $\mathrm{read} /$ write $(\mathrm{R} / \mathrm{W})$ electromagnetic transducers that are mounted on self-lubricated sub-micron flying slider bearings. These $\mathrm{R} / \mathrm{W}$ heads are connected to stainless steel suspension arms which are in turn connected to voice-coil actuators allowing cross-track seek and track-following motions.

Whereas bit density (along the track) is much higher than that of optical recording, track density of magnetic recording is typically much lower (although the access speed and data rate are much faster). Unlike optical recording, in magnetic recording, in order 


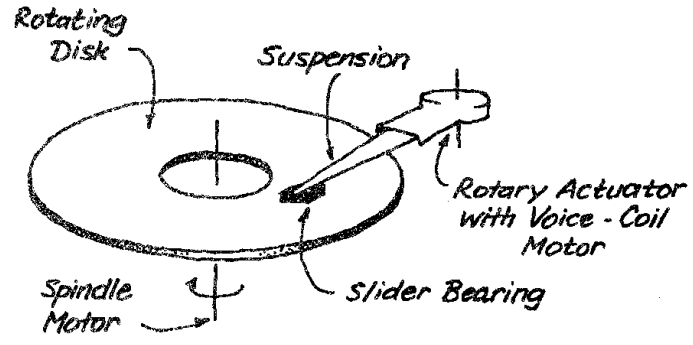

Figure 1: Major electromechanical components of a conventional high-performance rigid disk drive.

to maintain high bit density, the $\mathrm{R} / \mathrm{W}$ heads must be placed very close to the recording media. On the other hand, in order to minimize latency and maximize data transfer rate, the relative speed between the transducers and the media must be very high. Therefore, tribomechanical and tribochemical wear are important problems inherent in any form of magnetic recording and in order to maintain proper head/media interface, the actuator arm/suspension which serves as the interconnect between the actuator and the sensor must be very compliant in the vertical, pitch and roll directions. Unfortunately, such mechanical flexibilities tend to limit the bandwidth of the servo systems and therefore the track density. Furthermore, access time is severly limited by residual vibrations caused by the flexibility of the actuator-arm/suspension system, imposing another constraint on track density.

\section{Control Challenges}

Table 1 shows the approximate maximum areal density (MAD) of some typical commercial products of recent years as well as a projection of the required performance for products in the next decade. Note that areal data density is a combination of track density in the radial direction measured in tracks-perinch (TPI) and bit density in the tangential direction measured in bits-per-inch (BPI).

It is interesting to observe that historically BPI is at least an order of magnitude higher than TPI, resulting in a more than 20 to 1 aspect ratio in the lateral dimensions of the unit recording bit cell. It is also interesting to note that for the last two and a half decades, the industry has progressed on a steady $10 \mathrm{X} /$ decade $(+26 \%$ annual compound growth) improvement curve but since the early 90 's, in order to stay competitive, it has accelerated onto a $+60 \%$ annual growth curve. As a result, by the beginning of the next century, it is projected that the track density

\begin{tabular}{|c|c|c|c|}
\hline & MAD & TPI & BPI \\
\hline \hline $1971:$ & 1 & 200 & 5,000 \\
$1981:$ & 10 & 800 & 12,500 \\
$1991:$ & 100 & 2,000 & 50,000 \\
1995: & 600 & 5,000 & 120,000 \\
\hline \hline $2001:$ & 10,000 & 25,000 & 400,000 \\
\hline \hline $2005:$ & 100,000 & 100,000 & $1,000,000$ \\
\hline
\end{tabular}

Table 1: Approximate maximum areal density (measured in megabits per square inch) of recent commercial products and projections for the next decade.

will have to be at least 25,000 TPI which corresponds to a track pitch of $1 \mu \mathrm{m}$ and a tracking accuracy of less than $100 \mathrm{~nm}$. Furthermore, in another five years, it is projected that the magnetic recording industry must deliver at least 100,000 TPI which corresponds to a track pitch of $250 \mathrm{~nm}$ and an astonishing servo resolution of $25 \mathrm{~nm}$.

Besides the obvious contributing factors such as mechanical resonances, spindle runouts, temperature drifts, humidity variations, external shocks and vibrations, bearing hysteresis, cable bias, etc., there are a number of significant sources of off-track errors that are unique to computer disk drives. As opposed to other applications where cost is not a primary concern (aerospace, for example), in disk drives sensors are used only when they are absolutely necessary, otherwise the industry cannot remain cost competitive. Instead of using position sensors and servo patterns physically encoded on the disk surface, typically in computer disk drives off-track errors are derived using the so-called embedded servo technique where the necessary off-track informations are sandwiched within the magnetic data.

As the disk rotates, the magnetic $R$ / $W$ head will cross specially-coded magnetic servo patterns at regular intervals. After amplification, on-board demodulation electronics will convert the encoded servo data into an off-track position error signal. This signal is then fed into a digital servo controller which will compensate the error signal by driving the voice-coil actuator. Therefore, any non-linearity and inaccuracy in the servo patterns caused by defects in and inadequacy of the media, head and electronics will show up as measurable off-track error which cannot be avoided. As bit density and track density increase, this becomes more and more problematic.

In addition, besides track following, the servo system must also move the entire head/suspension assembly across the disk surface as fast as possible. This must 
be done in such a way to minimize residual vibration. Typically the servo controller will drive the actuator to follow an idealized velocity profile calculated based on a rigid body model. The actuator velocity is not measured since not only is there not a position sensor, there is also not a velocity sensor. Instead, the velocity is estimated from the embedded off-track error using a reduced-order Kalman-type estimator. Therefore, seek settling of the actuator/suspension system is one of the biggest source of off-track error (both residual vibration of the inherently flexible mechanical structures and the unavoidable velocity estimation error).

Obviously, the higher the servo bandwdith, the easier it is to correct these errors; and the higher the track density, the more important it is to have highbandwidth servo system. Currently, track density is less than 5,000 and servo bandwidth is approximately $500 \mathrm{~Hz}$ which is limited by inherent in-theloop mechanical resonances. For 25,000 TPI, it is projected that the servo bandwidth must be higher than $2 \mathrm{KHz}$. Furthermore, with spindle speed increased to 7,200 RPM, the required bandwidth will increase to 3 or $4 \mathrm{KHz}$. It is well recognized throughout the industry that this level of performance simply cannot be achieve by an evolution of the existing hardware. The only solution is to use a dual-stage piggyback actuator system similar to that of the optical disk drives. By moving only the $\mathrm{R} / \mathrm{W}$ head and not the entire slider/gimbal assembly, it is possible to design a high-bandwidth system without requiring unreasonable amount of power.

Aside from the aforementioned tracking control problems which mainly have to do with microactuation, there are other major technology issues pertinent to future-generation super-compact disk drives, for example, mechanical packaging, manufacturing and electromechanical interconnect. To be relevant to disk drive applications, any proposed microactuator design must be consistent with these concerns. In today's drive design, much of these problems are solved by compromising and synergizing the designs of the individual components, the $\mathrm{R} / \mathrm{W}$ head, the stainless steel suspension and the voice-coil motor.

Therefore, from the perspective of a disk drive system, one cannot design the microactuator without worrying about how it will affect the rest of the interconnecting components. Moreover, one cannot design it without thinking about how it will preserve the traditional volumetric cost advantage (dollar-permegabyte) of magnetic over optical disk drives (i.e., low disk-to-disk spacing, low media cost and low unit cost of electromechanical components). Finally, one must also project how the proposed design will coin- cide with the miniaturization trend of the magnetic disk drive industry such that it can lend itself to manufacturing automation and high-shock environments.

\section{Silicon Microstructures}

Conventional suspensions are made of stainless steel of uniform thickness with the various features manufactured by a combination of chemical etching, precision stamping and metal forming operations. As the drive form factor reduces to less than 2 inches in diameter and the slider bearing shrinks to about 1 by $1 \mathrm{~mm}$ in size, the suspension will be less than $1 \mathrm{~cm}$ long and the size of its gimbal (which is the structural component that connects the slider to the load-beam of the suspension) will be less than 1.5 $\mathrm{mm}$ square. Therefore, it becomes increasingly difficult if not altogether impossible to fabricate the suspension/gimbal system using existing material and manufacturing techniques.

Typically, one thinks of silicon as a brittle material and would not normally use it to construct flexible structures. However, brittle material is also elastic and the only difference is that unlike ductile material, it will not yield; it will simply fracture when the stress reaches the ultimate strength. It is well known that ultimate strength of silicon microstructures is orders of magnitude higher than that in bulk form. Therefore, silicon microsuspension can be made to be flexible but with a very high load-carrying capacity.

Figure 2 shows our proposed silicon microgimbal which has a number of potential operational advantages. First of all, since silicon is a brittle material, silicon microgimbals cannot be plastically deformed during manufacturing and handling; quality assurance therefore consists of only visual inspection. In comparison, plastic deformation of stainless steel suspensions is the major contributing factor of reduced production yield and in-drive performance problems, representing a significant cause of revenue loss.

In addition, due to the difference in process technology, much smaller features can be realized in silicon than in stainless steel (using various anisotropic wet and dry etching techniques), providing much needed additional degrees of design freedom. For example, in our design, we fabricate very intricate serpentine planar microsprings at the four corners of the microgimbal in order to satisfy the conflicting out-ofplane compliance, lateral stiffness and shock resistance design requirements.

Furthermore, with silicon, it is relatively easy to incorporate electrodes (or even pre-amps) in the gimbal 


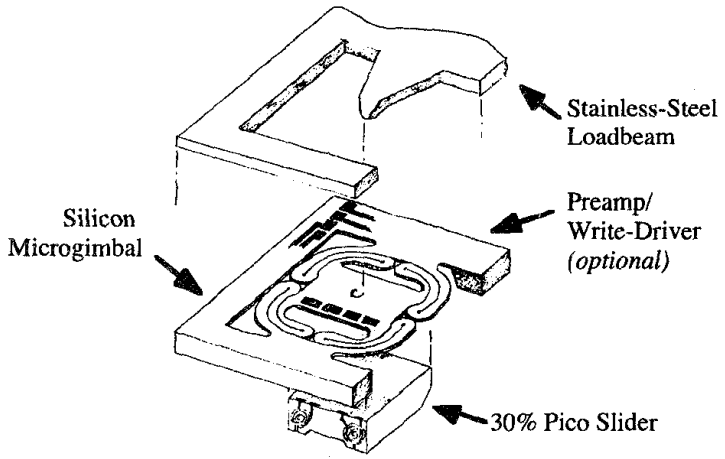

Figure 2: The proposed design concept of a hybrid silicon microgimbal/stainless steel suspension with built-in electrodes.

springs as well as an electrical coupler in the bonding area, thereby allowing the possibility of automating not only the mechanical but also the electrical interconnect manufacturing procedures. Currently, in many state-of-the-art products, assembly of the head/gimbal/suspension subsystem represents nearly half of the total manual labor cost.

The design requirements of the silicon microgimbals are quite challenging. In addition to being soft enough for head/media compliance and stiff enough for tracking (in order to increase servo bandwidth), they must also be strong enough to survive shocks on the order of hundreds of g's. In a way, designing microgimbals for the read/write heads is not much different from designing suspensions for our cars; automobile suspensions must be soft enough to give a smooth ride but yet stiff enough to handle fast turns. But most importantly, they must survive shocks with amplitudes well above normal operating conditions.

All suspension springs basically are composed of cantilever beams. The design trick of course, is to utilize cantilever beams of enough length such that for a given tip displacement, the operating strains are well below the yield or fracture point of the material. For applications where space is a concern, the obvious solution is to use helical springs which pack the most material into the smallest area. In the case of the microgimbal, the ideal solution would be to put individual helical springs at the four corners of the slider. Obviously, these soft springs must be designed such that they have sufficient stiffness in the lateral directions which requires careful optimization of the geometry (aspect ratio, radius, etc.).

Given the form factor that we are designing for, it is nearly impossible to design helical springs out of stainless steel sheet. On the other hand, the advantage of using silicon is that very small features can be achieved. The difficulty however, is not so much in fabricating a planar helical spring but in fabricating the interconnect between the center of the spring and the slider (or the substrate).

Figure 3 shows the individual planar microsprings at the four corners of the microgimbals and Figure 4 shows a summary of the processing steps. These springs are a variation of the conventional helical springs. Instead of spiral beams (which will require interconnects), they are composed of alternating curved beams. Much work have been performed in optimizing the various beam widths and the stress relief holes around the turns.

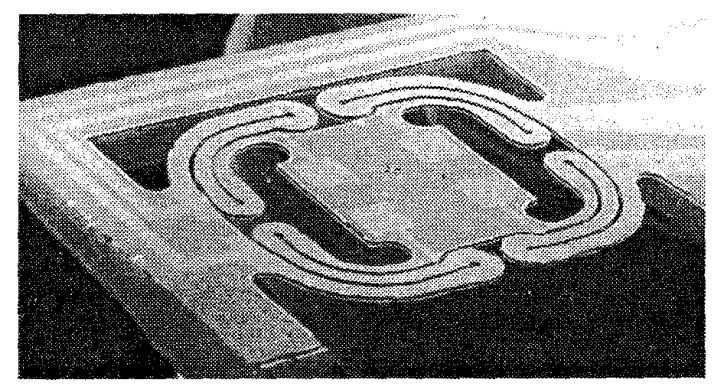

Figure 3: SEM micrograph of a three-turn silicon microgimbal.

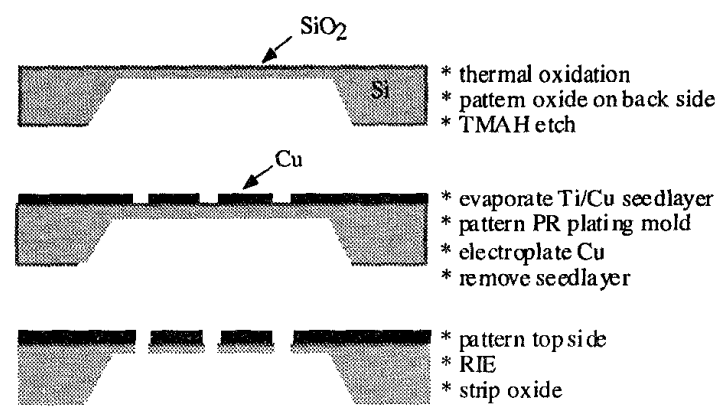

Figure 4: Processing steps for the silicon serpentine microgimbal.

\section{Silicon Microactuators}

Our next objective is to design and fabricate a piggyback microactuator which can be packaged to move the slider relative to the suspension such as to completely eliminate any in-the-loop structural resonances. This device must be very low-profile so that it is consistent with the disk-to-disk spacing requirement of future products. In addition, it must be massmanufacturable and be able to operate in low-voltage, 
low-power environment. Furthermore, electrical interference must be at an absolute minimum in order not to degrade the read/write operations.

Figure 5 shows a design concept which uses the aforementioned silicon microgimbal as the structural platform. Instead of a solid coupon in the center, the read/write slider is now attached to the microgimbal by a pair of hair-pin-like planar springs which are designed to have maximum in-plane compliance while maintaining adequate out-of-plane, pitch and roll stiffnesses.

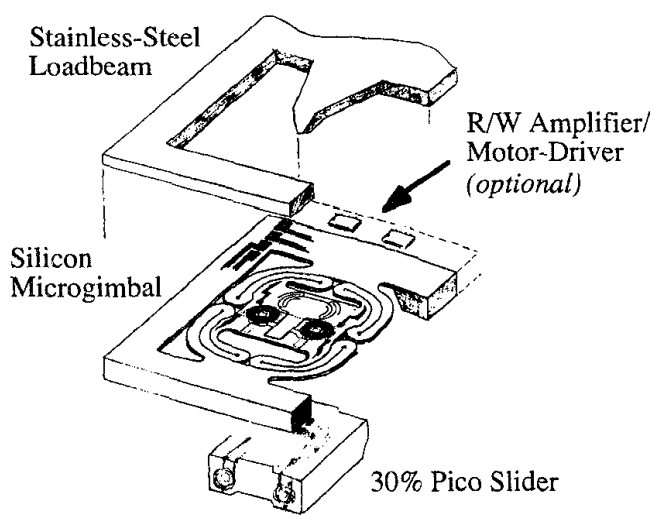

Figure 5: Design concept for a silicon micromachined electromagnetic piggyback planar microactuator for high TPI applications.

A pair of thin-film variable-reluctance microactuators are used to move the slider/recording head in the in-plane direction, each of which is consisted of two pieces. The stationary piece is mounted on the outside frame and is constructed of two layers of permalloy with a planar helical flat copper coil sandwiched in between. The moving permalloy piece is attached to the slider and when a voltage is applied to the coil, magnetic force will be exerted on the moving core, pulling it towards the stator and resulting in in-plane/cross-track micron-level fine motion of the read/write slider. This design has the important advantage that the requiired fabrication technology is very similar to that of thin-film heads, leveraging the expertise of the magnetic recording industry. Figure 6 summaries the fabrication process and Figure 7 shows a fabricated prototype.

\section{Summary}

Silicon micromachining techniques offer many exciting opportunities for fabricating both passive microstructures and active electromagnetic microactua-

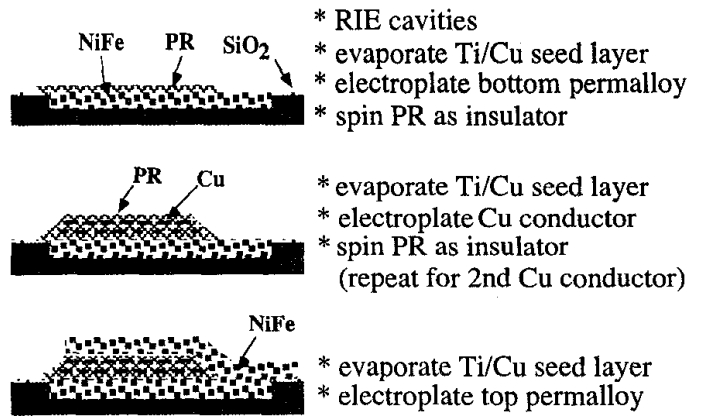

Figure 6: Process steps for the silicon microactuator.

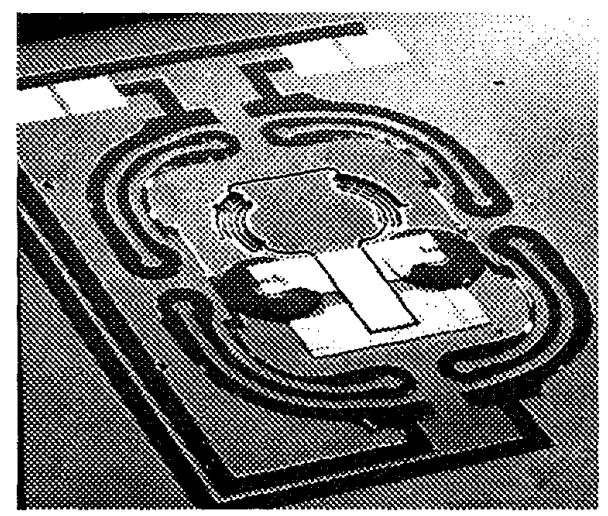

Figure 7: Micrograph of a fabricated prototype.

tors for significant form factor reduction and increase in recording density of future magnetic recording rigid disk drives. In this overview paper, we have presented some recent results and novel product concepts. In other related papers, we have documented detail results related to design optimization, process refinement and static/dynamic testing. ${ }^{1}$
${ }^{1}$ See Miu and Tai, "Silicon Micromachined SCALED Technology," 1993 JSME Conf. on Advanced Mechatronics, pp. 527-532; Miu et al., "Silicon Micromachined Microstructures for Super-Compact Magnetic Recording Rigid Disk Drives," Transducer '93 Conf., pp. 771-773; Miu et al., "Silicon Microgimbals for Super-Compact Magnetic Recording Rigid Disk Drives," ASME Advances in INFORMATION STORAGE SYSTEMS, Vol. 5, pp. 149-152; Temesvary et al., "Design, Fabrication and Testing of Silicon Microgimbals for Super -Compact Rigid Disk Drives," IEEE/ASME J. OF MEMS, March 1995; and Wu et al., "Silicon Micromachined Integrated Suspension Systems for 30\% Read/Write Pico-Sliders," 1994 ASME Winter Annual Meeting. 